\title{
The First Integral Method for Solving Maccari’s System
}

\author{
Davood Rostamy ${ }^{1,2}$, Fatemeh Zabihi ${ }^{1}$, Kobra Karimi ${ }^{1}$, Siamak Khalehoghli ${ }^{2}$ \\ ${ }^{1}$ Department of Mathematics, Imam Khomeini International University, Qazvin, Iran \\ ${ }^{2}$ Department of Mathematics, Islamic Azad University, South Tehran Branch, Tehran, Iran \\ E-mail: rostamyd@yahoo.com \\ Received November 13, 2010; revised December 31, 2010; accepted January 4, 2011
}

\begin{abstract}
In this paper, we investigate the first integral method for solving the solutions of Maccari's system. This idea can obtain some exact solutions of this system based on the theory of Commutative algebra.
\end{abstract}

Keywords: First Integral Method, Maccari’s System, Exact Solution

\section{Introduction}

The first integral method was first proposed for solving Burger-KdV equation [1] which is based on the ring theory of commutative algebra. This method was further developed by the same author in [2-10] and some other mathematicians $[2,11,12,13]$. The present paper investigates for the first time the applicability and effectiveness of the first integral method on the Maccari's. We consider Maccari's system:

$$
\left\{\begin{array}{l}
i Q_{t}+Q_{x x}+Q R=0, \\
R_{t}+R_{y}+\left(|Q|^{2}\right)_{x}=0 .
\end{array}\right.
$$

For the first time, Maccari derived this system from the Kadomtsev-Petviashvili equation by using asymptotically exact reduction method based Fourier expansion and spatiotemporal rescaling [14]. Maccari's system is a kind of nonlinear evolution equations that are often presented to describe the motion of the isolated waves, localized in a small part of space, in many fields such as hydrodynamic, plasma physics, nonlinear optic, etc. Zhang used Exp-function method for seeking exact solutions of Maccari's system [15].

The remaining structure of this article is organized as follows: Section 2 is a brief introduction to the first integral method. In Section 3, implementing the first integral method, some new exact solutions for Maccari's system are reported. This describes ability and reliability of the method. A conclusion and future directions for research are all summarized in the last section.

\section{The First Integral Method}

Consider a general nonlinear PDE in the form

$$
P\left(u, u_{t}, u_{x}, u_{y}, u_{x x}, u_{t t}, u_{y y}, u_{x t}, u_{x y}, u_{y t}, u_{x x x}, \cdots\right)=0 .
$$

Using the wave variable $\eta=x+\beta y-2 k t$ carries into the following ODE:

$$
Q\left(U, U^{\prime}, U^{\prime \prime}, U^{\prime \prime \prime}, \cdots\right)=0,
$$

where prime denotes the derivative with respect to the same variable $\eta$.

Next, we introduce new independent variables $x=u$, $y=u_{\eta}$ which change to a system of ODEs

$$
\left\{\begin{array}{l}
x^{\prime}=y \\
y^{\prime}=f(x, y) .
\end{array}\right.
$$

According to the qualitative theory of differential equations [1], if one can find two first integrals to system (4) under the same conditions, then analytic solutions to (4) can be solved directly. However, in general, it is difficult to realize this even for a single first integral, because for a given plane autonomous system, there is no general theory telling us how to find it's first integrals in a systematic way. A key idea of our approach here to find first integral is to utilize the division theorem. For convenience, first let us recall the division theorem for two variables in the complex domain $\mathbb{C}$ [4].

Theorem 2.1. Division theorem (see [14]) Suppose that $P(x, y)$ and $Q(x, y)$ are polynomials of two variables $x$ and $y$ in $\mathbb{C}[x, y]$ and $P(x, y)$ is irreducible in $\mathbb{C}[x, y]$. If $Q(x, y)$ vanishes at all zero points of $P(x, y)$, then there exists a polynomial $G(x, y)$ in $\mathbb{C}[x, y]$ such that $Q(x, y)=P(x, y) G(x, y)$.

\section{Exact Solutions for Maccari’s System}

In order to seek exact solutions of system (1), we sup- 
pose

$$
Q(x, y, t)=u(x, y, t) \exp [i(k x+\alpha y+\lambda t+l)],
$$

where $k, \alpha$ and $\lambda$ are constants to be determined later, $l$ is an arbitrary constant. Substituting Equation (5) into system (1) and yields

$$
\left\{\begin{array}{l}
i\left(u_{t}+2 k u_{x}\right)+u_{x x}-\left(\lambda+k^{2}\right) u+u R=0, \\
R_{t}+R_{y}+\left(u^{2}\right)_{x}=0,
\end{array}\right.
$$

Using the transformation

$$
u=u(\eta), R=R(\eta), \eta=x+\beta y-2 k t,
$$

where $\beta$ is a constant, system (6) become the following

$$
\left\{\begin{array}{l}
u^{\prime \prime}-\left(\lambda+k^{2}\right) u+u R=0, \\
(\beta-2 k) R^{\prime}+\left(u^{2}\right)^{\prime}=0,
\end{array}\right.
$$

where prime denotes the differential with respect to $\eta$. Integrating the second segment of Equation (8) with respect to $\eta$ and taking the integration constant as zero yields yields

$$
R=\frac{-1}{\beta-2 k} u^{2}
$$

Substituting Equation (9) into the first segment of (8) yields

$$
u^{\prime \prime}-\left(\lambda+k^{2}\right) u-\frac{1}{\beta-2 k} u^{3}=0 .
$$

Next, we introduce new independent variables $x=u$, $y=u_{\eta}$ which change Equation (10) to a system of ODEs

$$
\left\{\begin{array}{l}
x^{\prime}=y \\
y^{\prime}=\left(\lambda+k^{2}\right) x+\frac{1}{\beta-2 k} x^{3} .
\end{array}\right.
$$

Now, we are applying the Division Theorem to seek the first integral to (9). Suppose that $x=x(\eta)$ and $y=y(\eta)$ are the nontrivial solutions to (9), and

$$
p(x, y)=\sum_{i=0}^{m} a_{i}(x) y^{i},
$$

is an irreducible polynomial in $\mathbb{C}[x, y]$ such that

$$
p(x(\eta), y(\eta))=\sum_{i=0}^{m} a_{i}(x(\eta)) y(\eta)^{i}=0,
$$

where $a_{i}(x)(i=0,1, \cdots, m)$ are polynomials of $x$ and all relatively prime in $\mathbb{C}[x, y], a_{m}(x) \neq 0$. Equation (10) is also called the first integral to (9). We start our study by assuming $m=1$ in (12). Note that $\frac{d p}{d \eta}$ is a polynomial in $x$ and $y$, and $p[x(\eta), y(\eta)]=0$ implies $\left.\frac{d p}{d \eta}\right|_{(11)}=0$. By the Division Theorem, there exists a polynomial $H(x, y)=h(x)+g(x) y$ in $\mathbb{C}[x, y]$ such that

$$
\begin{aligned}
& \left.\frac{d p}{d \eta}\right|_{(11)}=\left.\left(\frac{\partial p}{\partial x} \frac{\partial x}{\partial \eta}+\frac{\partial p}{\partial y} \frac{\partial y}{\partial \eta}\right)\right|_{(11)} \\
& =\sum_{i=0}^{1} a_{i}^{\prime}(x) y^{i+1}+\sum_{i=0}^{1} i a_{i}(x) y^{i-1}\left(\left(\lambda+k^{2}\right) x+\frac{1}{\beta-2 k} x^{3}\right) \\
& =(h(x)+g(x) y)\left(\sum_{i=0}^{1} a_{i}(x) y^{i}\right),
\end{aligned}
$$

where prime denotes differentiating with respect to the variable $x$. On equating the coefficients of $y^{i}(i=2,1,0)$ on both sides of (13), we have

$$
\begin{gathered}
a_{1}^{\prime}(x)=g(x) a_{1}(x), \\
a_{0}^{\prime}(x)=h(x) a_{1}(x)+g(x) a_{0}(x), \\
a_{1}(x)\left(\left(\lambda+k^{2}\right) x+\frac{1}{(\beta-2 k)} x^{3}\right)=h(x) a_{0}(x) .
\end{gathered}
$$

Since, $a_{1}(x)$ is a polynomial of $x$, from (12) we conclude that $a_{1}(x)$ is a constant and $g(x)=0$. For simplicity, we take $a_{1}(x)=1$, and balancing the degrees of $h(x)$ and $a_{0}(x)$ we conclude that deg $h(x)=1$, only. Now suppose that $h(x)=A x+B$, then From (13), we find

$$
a_{0}(x)=\frac{1}{2} A x^{2}+B x+D,
$$

where $D$ is an arbitrary integration constant. Substituting $a_{0}(x), a_{1}(x)$ and $h(x)$ in (14) and setting all the coefficients of powers $x$ to be zero, we obtain a system of nonlinear algebraic equations and by solving it, we obtain the following solutions:

$$
A=\frac{\sqrt{2}}{\sqrt{\beta-2 k}}, B=0, D=\frac{\sqrt{2}}{2}\left(\lambda+k^{2}\right) \sqrt{\beta-2 k},
$$

and

$$
A=-\frac{\sqrt{2}}{\sqrt{\beta-2 k}}, B=0, D=-\frac{\sqrt{2}}{2}\left(\lambda+k^{2}\right) \sqrt{\beta-2 k} .
$$

Using (15) and (16) in (10), we obtain

$$
y+\frac{\sqrt{2}}{2 \sqrt{\beta-2 k}} x^{2}+\frac{\sqrt{2}\left(\lambda+k^{2}\right) \sqrt{\beta-2 k}}{2}=0,
$$

and

$$
y-\frac{\sqrt{2}}{2 \sqrt{\beta-2 k}} x^{2}-\frac{\sqrt{2}\left(\lambda+k^{2}\right) \sqrt{\beta-2 k}}{2}=0,
$$

respectively. Combining this equations with (9), we obtain the exact solutions of Equation (10) as follows: 


$$
\begin{aligned}
& u_{1}(\eta)=\sqrt{2 k-\beta} \sqrt{\lambda+k^{2}} \tanh \left(-\frac{\sqrt{2}}{2} i \sqrt{\lambda+k^{2}} \eta-\sqrt{2 k-\beta} \sqrt{\lambda+k^{2}} c_{1}\right), \\
& u_{2}(\eta)=\sqrt{2 k-\beta} \sqrt{\lambda+k^{2}} \tanh \left(\frac{\sqrt{2}}{2} i \sqrt{\lambda+k^{2}} \eta-\sqrt{2 k-\beta} \sqrt{\lambda+k^{2}} c_{1}\right)
\end{aligned}
$$

where $c_{1}$ is an arbitrary constant. Therefore, the exact solutions to (10) can be written as

$$
\begin{aligned}
& u_{1}(x, y, t)=\sqrt{2 k-\beta} \sqrt{\lambda+k^{2}} \tanh \left(-\frac{\sqrt{2}}{2} i \sqrt{\lambda+k^{2}}(x+\beta y-2 k t)-\sqrt{2 k-\beta} \sqrt{\lambda+k^{2}} c_{1}\right), \\
& u_{2}(x, y, t)=\sqrt{2 k-\beta} \sqrt{\lambda+k^{2}} \tanh \left(\frac{\sqrt{2}}{2} i \sqrt{\lambda+k^{2}}(x+\beta y-2 k t)-\sqrt{2 k-\beta} \sqrt{\lambda+k^{2}} c_{1}\right) .
\end{aligned}
$$

Then exact solutions for system (1) are

$$
\left\{\begin{array}{l}
R_{1}=-\left(\lambda+k^{2}\right) \tanh ^{2}\left(-\frac{\sqrt{2}}{2} i \sqrt{\lambda+k^{2}}(x+\beta y-2 k t)-\sqrt{2 k-\beta} \sqrt{\lambda+k^{2}} c_{1}\right) \\
Q_{1}=e^{i(k x+\alpha y+\lambda t+l)} \sqrt{2 k-\beta} \sqrt{\lambda+k^{2}} \tanh \left(-\frac{\sqrt{2}}{2} i \sqrt{\lambda+k^{2}}(x+\beta y-2 k t)-\sqrt{2 k-\beta} \sqrt{\lambda+k^{2}} c_{1}\right) .
\end{array}\right.
$$

and

$$
\left\{\begin{array}{l}
R_{2}=-\left(\lambda+k^{2}\right) \tanh ^{2}\left(\frac{\sqrt{2}}{2} i \sqrt{\lambda+k^{2}}(x+\beta y-2 k t)-\sqrt{2 k-\beta} \sqrt{\lambda+k^{2}} c_{1}\right), \\
Q_{2}=e^{i(k x+\alpha y+\lambda t+l)} \sqrt{2 k-\beta} \sqrt{\lambda+k^{2}} \tanh \left(\frac{\sqrt{2}}{2} i \sqrt{\lambda+k^{2}}(x+\beta y-2 k t)-\sqrt{2 k-\beta} \sqrt{\lambda+k^{2}} c_{1}\right) .
\end{array}\right.
$$

Now we assume that $m=2$ in (10). By the Division Theorem, there exists a polynomial $H(x, y)=h(x)+g(x) y$ in $\mathbb{C}[x, y]$ such that

$$
\begin{aligned}
& \left.\frac{d p}{d \eta}\right|_{(11)}=\left.\left(\frac{\partial p}{\partial x} \frac{\partial x}{\partial \eta}+\frac{\partial p}{\partial y} \frac{\partial y}{\partial \eta}\right)\right|_{(11)} \\
& =\sum_{i=0}^{2} a_{i}^{\prime}(x) y^{i+1}+\sum_{i=0}^{2} i a_{i}(x) y^{i-1}\left(\left(\lambda+k^{2}\right) x+\frac{1}{\beta-2 k} x^{3}\right) \\
& =(h(x)+g(x) y)\left(\sum_{i=0}^{2} a_{i}(x) y^{i}\right) .
\end{aligned}
$$

On equating the coefficients of $y^{i}(i=3,2,1,0)$ on both sides of (21), we have

$$
\begin{gathered}
a_{2}^{\prime}(x)=g(x) a_{2}(x), \\
a_{1}^{\prime}(x)=h(x) a_{2}(x)+g(x) a_{1}(x), \\
a_{0}^{\prime}(x)=-2 a_{2}(x)\left(\left(\lambda+k^{2}\right) x+\frac{1}{(\beta-2 k)} x^{3}\right) \\
+h(x) a_{1}(x)+g(x) a_{0}(x), \\
a_{1}(x)\left(\left(\lambda+k^{2}\right) x+\frac{1}{(\beta-2 k)} x^{3}\right)=h(x) a_{0}(x) .
\end{gathered}
$$

Since, $a_{2}(x)$ is a polynomial of $x$, from (20) we conclude that $a_{2}(x)$ is a constant and $g(x)=0$. For simplicity, we take $a_{2}(x)=1$, and balancing the degrees of $h(x), a_{0}(x)$ and $a_{1}(x)$ we conclude that deg $h(x)=1$ or 0 , therefore we have two cases:

\section{Case1:}

Suppose that deg $h(x)=1$ and $h(x)=A x+B$, then from (21) we find

$$
a_{1}(x)=\frac{1}{2} A x^{2}+B x+D,
$$

where $D$ is an arbitrary integration constant. From (22) we find

$$
\begin{aligned}
a_{0}(x) & =\left(\frac{A^{2}}{8}-\frac{1}{2(\beta-2 k)}\right) x^{4}+\frac{A B}{2} x^{3} \\
& +\left(\frac{1}{2}\left(A D+B^{2}-2\left(\lambda+k^{2}\right)\right)\right) x^{2}+B D x+E,
\end{aligned}
$$

where $E$ is an arbitrary integration constant. Substituting $a_{0}(x), a_{1}(x)$ and $h(x)$ in (25) and setting all the coefficients of powers $x$ to be zero, we obtain a system of nonlinear algebraic equations and by solving it, we obtain

$$
\begin{aligned}
& E=\frac{\left(\lambda+k^{2}\right)^{2}(\beta-2 k)}{2}, \quad B=0, \\
& D=\left(\lambda+k^{2}\right) \sqrt{2(\beta-2 k)}, \\
& A=\frac{2 \sqrt{2}}{\sqrt{\beta-2 k}},
\end{aligned}
$$


and

$$
\begin{aligned}
& E=\frac{\left(\lambda+k^{2}\right)^{2}(\beta-2 k)}{2}, \\
& B=0, \\
& D=-\left(\lambda+k^{2}\right) \sqrt{2(\beta-2 k)}, \\
& A=-\frac{2 \sqrt{2}}{\sqrt{\beta-2 k}} .
\end{aligned}
$$

Using (26) and (25) in (10), we obtain

$$
y+\frac{1}{\sqrt{2(\beta-2 k)}} x^{2}+\frac{\left(\lambda+k^{2}\right) \sqrt{\beta-2 k}}{\sqrt{2}}=0,
$$

and

$$
y-\frac{1}{\sqrt{2(\beta-2 k)}} x^{2}-\frac{\left(\lambda+k^{2}\right) \sqrt{\beta-2 k}}{\sqrt{2}}=0,
$$

respectively. Combining this Equations with (11), we obtain two exact solutions to Equation (10) which was obtained in case $\mathrm{m}=1$, i.e.

$$
\begin{aligned}
& u_{1}(\eta)=\sqrt{2 k-\beta} \sqrt{k^{2}+\lambda} \tanh \left(-\frac{\sqrt{2}}{2} i \sqrt{k^{2}+\lambda} \eta-\sqrt{2 k-\beta} \sqrt{k^{2}+\lambda} c_{1}\right), \\
& u_{2}(\eta)=\sqrt{2 k-\beta} \sqrt{k^{2}+\lambda} \tanh \left(\frac{\sqrt{2}}{2} i \sqrt{k^{2}+\lambda} \eta-\sqrt{2 k-\beta} \sqrt{k^{2}+\lambda} c_{1}\right) .
\end{aligned}
$$

where $c_{1}$ is an arbitrary constant. Therefore, the exact solutions to (10) can be written as

$$
\begin{aligned}
& u_{1}(x, y, t)=\sqrt{2 k-\beta} \sqrt{\lambda+k^{2}} \tanh \left(-\frac{\sqrt{2}}{2} i \sqrt{\lambda+k^{2}}(x+\beta y-2 k t)-\sqrt{2 k-\beta} \sqrt{\lambda+k^{2}} c_{1}\right), \\
& u_{2}(x, y, t)=\sqrt{2 k-\beta} \sqrt{\lambda+k^{2}} \tanh \left(\frac{\sqrt{2}}{2} i \sqrt{\lambda+k^{2}}(x+\beta y-2 k t)-\sqrt{2 k-\beta} \sqrt{\lambda+k^{2}} c_{1}\right) .
\end{aligned}
$$

Then the exact solutions for system (1) are:

$$
\left\{\begin{array}{l}
R_{1}=-\left(\lambda+k^{2}\right) \tanh ^{2}\left(-\frac{\sqrt{2}}{2} i \sqrt{\lambda+k^{2}}(x+\beta y-2 k t)-\sqrt{2 k-\beta} \sqrt{\lambda+k^{2}} c_{1}\right), \\
Q_{1}=e^{i(k x+\alpha y+\lambda t+l)} \sqrt{2 k-\beta} \sqrt{\lambda+k^{2}} \tanh \left(-\frac{\sqrt{2}}{2} i \sqrt{\lambda+k^{2}}(x+\beta y-2 k t)-\sqrt{2 k-\beta} \sqrt{\lambda+k^{2}} c_{1}\right) .
\end{array}\right.
$$

and

$$
\left\{\begin{array}{l}
R_{2}=-\left(\lambda+k^{2}\right) \tanh ^{2}\left(\frac{\sqrt{2}}{2} i \sqrt{\lambda+k^{2}}(x+\beta y-2 k t)-\sqrt{2 k-\beta} \sqrt{\lambda+k^{2}} c_{1}\right), \\
Q_{2}=e^{i(k x+\alpha y+\lambda t+l)} \sqrt{2 k-\beta} \sqrt{\lambda+k^{2}} \tanh \left(\frac{\sqrt{2}}{2} i \sqrt{\lambda+k^{2}}(x+\beta y-2 k t)-\sqrt{2 k-\beta} \sqrt{\lambda+k^{2}} c_{1}\right) .
\end{array}\right.
$$

\section{Case 2:}

In this case suppose that $\operatorname{deg} h(x)=0$ and $h(x)=A$, then from (21) we find $a_{1}(x)=A x+B$, where $B$ is an arbitrary integration constant. From (22) we find

$$
a_{0}(x)=\frac{-1}{2(\beta-2 k)} x^{4}+\left(\frac{A^{2}}{2}-\left(\lambda+k^{2}\right)\right) x^{2}+A B x+D,
$$

where $D$ is an arbitrary integration constant. Substituting $a_{0}(x), a_{1}(x)$ and $h(x)$ in (23) and setting all the coefficients of powers $x$ to be zero, we obtain a system of nonlinear algebraic equations and by solving it, we obtain

$$
A=0, B=0 .
$$

Using (28) in (10), we obtain

$$
y^{2}-\left(\lambda+k^{2}\right) x^{2}-\frac{1}{2(\beta-2 k)} x^{4}=0 .
$$

Combining this equations with (9), we obtain the exact solutions to Equation (10) as follows:

$$
\begin{aligned}
& u_{3}(\eta)=\frac{4 \sqrt{2} \sqrt{\beta-2 k} \sqrt{\lambda+k^{2}} e^{\left(\sqrt{2} c_{1} \sqrt{\beta-2 k} \sqrt{\lambda+k^{2}}-\eta \sqrt{\lambda+k^{2}}\right)}}{-4 e^{2 \sqrt{2} c_{1} \sqrt{\beta-2 k} \sqrt{\lambda+k^{2}}}+e^{-2 \eta \sqrt{\lambda+k^{2}}}}, \\
& u_{4}(\eta)=\frac{4 \sqrt{2} \sqrt{\beta-2 k} \sqrt{\lambda+k^{2}} e^{\left(\sqrt{2} c_{1} \sqrt{\beta-2 k} \sqrt{\lambda+k^{2}}-\eta \sqrt{\lambda+k^{2}}\right)}}{1-4 e^{\left(2 \sqrt{2} c_{1} \sqrt{\beta-2 k} \sqrt{\lambda+k^{2}}-2 \eta \sqrt{\lambda+k^{2}}\right)}},
\end{aligned}
$$

where $c_{1}$ is an arbitrary constant. Then the exact solutions to (10) can be written as: 


$$
\begin{aligned}
& u_{3}(x, y, t)=\frac{4 \sqrt{2} \sqrt{\beta-2 k} \sqrt{\lambda+k^{2}} e^{\left(\sqrt{2} c_{1} \sqrt{\beta-2 k} \sqrt{\lambda+k^{2}}-(x+\beta y-2 k t) \sqrt{\lambda+k^{2}}\right)}}{-4 e^{2 \sqrt{2} c_{1} \sqrt{\beta-2 k} \sqrt{\lambda+k^{2}}}+e^{-2(x+\beta y-2 k t) \sqrt{\lambda+k^{2}}}}, \\
& u_{4}(x, y, t)=\frac{4 \sqrt{2} \sqrt{\beta-2 k} \sqrt{\lambda+k^{2}} e^{\left(\sqrt{2} c_{1} \sqrt{\beta-2 k} \sqrt{\lambda+k^{2}}-(x+\beta y-2 k t) \sqrt{\lambda+k^{2}}\right)}}{1-4 e^{\left(2 \sqrt{2} c_{1} \sqrt{\beta-2 k} \sqrt{\lambda+k^{2}}-2(x+\beta y-2 k t) \sqrt{\lambda+k^{2}}\right)}},
\end{aligned}
$$

Then solutions of system (1) are

$$
\left\{\begin{array}{l}
R_{3}=\frac{-32\left(\lambda+k^{2}\right) e^{2\left(\sqrt{2} c_{1} \sqrt{\beta-2 k} \sqrt{\lambda+k^{2}}-(x+\beta y-2 k t) \sqrt{\lambda+k^{2}}\right)}}{\left(-4 e^{2 \sqrt{2} c_{1} \sqrt{\beta-2 k} \sqrt{\lambda+k^{2}}}+e^{-2(x+\beta y-2 k t) \sqrt{\lambda+k^{2}}}\right)^{2}}, \\
Q_{3}=\frac{4 \sqrt{2} \sqrt{\beta-2 k} \sqrt{\lambda+k^{2}} e^{\left(\sqrt{2} c_{1} \sqrt{\beta-2 k} \sqrt{\lambda+k^{2}}-(x+\beta y-2 k t) \sqrt{\lambda+k^{2}}+i(k x+\alpha y+\lambda t+l)\right)}}{-4 e^{2 \sqrt{2} c_{1} \sqrt{\beta-2 k} \sqrt{\lambda+k^{2}}}+e^{-2(x+\beta y-2 k t) \sqrt{\lambda+k^{2}}}} .
\end{array}\right.
$$

and

$$
\left\{\begin{array}{l}
R_{4}=\frac{-32\left(\lambda+k^{2}\right) e^{2\left(\sqrt{2} c_{1} \sqrt{\beta-2 k} \sqrt{\lambda+k^{2}}-(x+\beta y-2 k t) \sqrt{\lambda+k^{2}}\right)}}{\left(1-4 e^{\left.\left(2 \sqrt{2} c_{1} \sqrt{\beta-2 k} \sqrt{\lambda+k^{2}}-2(x+\beta y-2 k t) \sqrt{\lambda+k^{2}}\right)\right)^{2}}\right.}, \\
Q_{4}=\frac{4 \sqrt{2} \sqrt{\beta-2 k} \sqrt{\lambda+k^{2}} e^{\left(\sqrt{2} c_{1} \sqrt{\beta-2 k} \sqrt{\lambda+k^{2}}-(x+\beta y-2 k t) \sqrt{\lambda+k^{2}}+i(k x+\alpha y+\lambda t+l)\right)}}{1-4 e^{\left(2 \sqrt{2} c_{1} \sqrt{\beta-2 k} \sqrt{\lambda+k^{2}}-2(x+\beta y-2 k t) \sqrt{\lambda+k^{2}}\right)}} .
\end{array}\right.
$$

\section{Conclusions}

We described this method for finding some new exact solutions for the Maccari's system. We have obtained four exact solutions to the Maccari's system. The solutions obtained are expressed by the hyperbolic and exponential functions. These new solutions may be important for the explanation of some practical physical problems. This also suggests that one can find different solutions by choosing different methods.

\section{Acknowledgements}

The support of Islamic Azad University of South Tehran Branch is gratefully acknowledged.

\section{References}

[1] Z. Feng, "On Explicit Exact Solutions to the Compound Burgers-KdV Equation,” Physics Letters A, Vol. 293, No. 1, 2002, pp. 57-66. doi:10.1016/S0375-9601(01)00825-8

[2] X. Deng, "Traveling Wave Solutions for the Generalized Burgers Huxley Equation,” Applied Mathematics and Computation, Vol. 204, No. 2, 2008, pp. 733-737. doi:10.1016/j.amc.2008.07.020
[3] R. Z. Feng and G. Chenb, "Solitary Wave Solutions of the Compound Burgers-Korteweg-de Vries Equation,” Physica A, Vol. 352, No. 2-4, 2005, pp. 419-435. doi:10.1016/j.physa.2004.12.061

[4] Z. Feng, "Traveling Wave Behavior for a Generalized Fisher Equation,” Chaos, Solitons \& Fractals, Vol. 38, No. 2, 2008, pp. 481-488. doi:10.1016/j.chaos.2006.11.031

[5] Z. Feng and Y. Li, "Complex Traveling Wave Solutions to the Fisher Equation,” Physica A, Vol. 366, 2006, pp. 115-123. doi:10.1016/j.physa.2005.10.058

[6] A. Z. Feng, "Exact Solution to an Approximate SineGordon Equation in $(\mathrm{n}+1)$-Dimensional Space," Physics Letters A, Vol. 302, No. 2-3, 2002, pp. 64-76. doi:10.1016/S0375-9601(02)01114-3

[7] Z. Feng and X. Wang, "The First Integral Method to the Two-Dimensional Burgers-Korteweg-de Vries Equation,” Physics Letters A, Vol. 308, No. 2-3, 2003, pp. 173-178. doi:10.1016/S0375-9601(03)00016-1

[8] Z. Feng and R. Knobel, "Traveling Waves to a BurgersKorteweg-de Vries-Type Equation with Higher-Order Nonlinearities," Journal of Mathematical Analysis and Applications, Vol. 328, No. 2, 2007, pp. 1435-1450. doi:10.1016/j.jmaa.2006.05.085

[9] H. Li and Y. Guo, "New Exact Solutions to the Fitzhugh-Nagumo Equation,” Applied Mathematics and Com- 
putation, Vol. 180, No. 2, 2006, pp. 524- 528.

doi:10.1016/j. amc.2005.12.035

[10] B. Lu, H. Zhang and F. Xie, "Traveling Wave Solutions of Nonlinear Partial Equations by Using the First Integral Method," Applied Mathematics and Computation, Vol. 216, No. 4, 2010, pp. 1329-1336.

doi:10.1016/j.amc.2010.02.028

[11] A. Maccari, "The Kadomtsev-Petviashvili Equation as a Source of Integrable Model Equations," Journal of Mathematical Physics, Vol. 37, 1996, pp. 5897-6590. doi:10.\1063/1.531773

[12] L. F. Tascan, A. Bekir and M. Koparan, "Travelling Wave Solutions of Nonlinear Evolution Equations by
Using the First Integral Method,” Communications in Nonlinear Science and Numerical Simulation, Vol. 14, No. 5, 2009, pp. 1810-1815. doi:10.1016/j.cnsns.2008.07.009

[13] Z. Feng, "On Explicit Exact Solutions to the Compound Burgers-KdV Equation,” Physics Letters A, Vol. 293, No. 1-2, 2002, pp. 57-66. doi:10.1016/S0375-9601(01)00825-8

[14] T S. Zhang, "Exp-Function Method for Solving Maccari’s System, Physics Letters A, Vol. 371, No. 1-2, 2007, pp. 65-71. doi:10.1016/j.physleta.2007.05.091

[15] T. R. Ding and C. Z. Li, “Ordinary Differential Equations,” Peking University Press, Peking, 1996. 